\title{
Studying collective nuclear excitations with fast neutrons: a comparison of ${ }^{76} \mathrm{Ge}$ and ${ }^{76} \mathrm{Se}$
}

\author{
Steven $W$. Yates ${ }^{1, *}$, Sharmistha Mukhopadhyay ${ }^{1}$, Benjamin P. Crider ${ }^{2}$, and Erin E. Peters ${ }^{1}$ \\ ${ }^{1}$ Departments of Chemistry and Physics \& Astronomy, University of Kentucky, Lexington, Kentucky, \\ 40506-0055, USA \\ ${ }^{2}$ Department of Physics and Astronomy, Mississippi State University, Mississippi State, Mississippi, \\ 39762, USA
}

\begin{abstract}
The low-lying, low-spin level structures of ${ }^{76} \mathrm{Ge}$ and ${ }^{76} \mathrm{Se}$ were studied with the $\left(n, n^{\prime} \gamma\right)$ reaction. Gamma-ray excitation function and angular distribution measurements were performed, and level spins, level lifetimes, $\gamma$-ray intensities, and multipole mixing ratios were determined. Large observed B(E2) values support the identification of low-lying band structures.
\end{abstract}

\section{Introduction}

There are many excellent reasons for studying the stable $\mathrm{A}=76$ nuclei in detail. In recent years, the most frequently expressed motivation is related to the possibility of observing neutrinoless double-beta decay $(0 v \beta \beta)$. Indeed, the two-neutrino double-beta decay $(2 v \beta \beta)$ of ${ }^{76} \mathrm{Ge}$, the parent, into ${ }^{76} \mathrm{Se}$, the daughter, has been observed, and ${ }^{76} \mathrm{Ge}$ is considered one of the best $0 v \beta \beta$ candidates [1]. The observation of $0 v \beta \beta$ would have profound implications as the neutrino would be exposed as its own antiparticle, lepton number non-conservation would be verified for the first time, and a new window would be opened for determining the mass of the neutrino. Three factors determine the $0 v \beta \beta$ decay rate: a phase space factor, which can be reliably calculated; the effective Majorana neutrino mass, for which only an upper limit is known; and the nuclear matrix element, NME [2]. The NME cannot be experimentally determined but must be obtained from nuclear structure theory. Unfortunately, comparisons of NMEs calculated with various theories significantly disagree, frequently by factors of two or three [2]. However, measurements of the structural characteristics and properties of ${ }^{76} \mathrm{Ge}$ and ${ }^{76} \mathrm{Se}$ can be used to constrain the theoretical calculations.

In addition to the reasons related to $0 v \beta \beta$, there are other goals for our studies. The $\mathrm{A}=$ 76 shape-transitional region is structurally interesting. Shape coexistence was observed many years ago [3, 4], and recent arguments about existence of rigid triaxiality have emerged $[5,6]$.

\footnotetext{
*Corresponding author: yates@uky.edu
} 


\section{Experimental methods}

\subsection{Inelastic neutron scattering}

Inelastic scattering of fast neutrons in the few $\mathrm{MeV}$ range followed by $\gamma$-ray detection can yield a variety of valuable spectroscopic quantities. At the University of Kentucky Accelerator Laboratory (UKAL), neutrons are produced by the ${ }^{3} \mathrm{H}(\mathrm{p}, \mathrm{n}){ }^{3} \mathrm{He}$ or ${ }^{2} \mathrm{H}(\mathrm{d}, \mathrm{n})^{3} \mathrm{He}$ reactions, and these fast, nearly monoenergetic $(\Delta \mathrm{E} \approx 50 \mathrm{keV})$ neutrons are used to excite the target nuclei.

There are a number of advantages of the (n,n' $\gamma)$ reaction.

- As neutrons are uncharged and experience no Coulomb barrier, the nucleus can be gently probed. Moreover, the degree of excitation can be selected because the incident neutron energy can be controlled by changing the energy of the incident charged particles.

- As $\gamma$ rays are detected, the energy resolution is excellent.

- At the neutron energies used in these measurements $(<4 \mathrm{MeV})$, the direct reaction contributions are generally regarded as small, and the compound nucleus assumption permits us to treat the population of levels statistically (i.e., non-selectively, but limited by angular momentum considerations).

- Lifetimes can be determined by the Doppler-shift attenuation method (DSAM) [7, 8].

- When required, $\gamma-\gamma$ coincidence measurements are possible $[9,10]$.

As with any method, there are limitations.

- Studies are generally limited to stable nuclei or those with long lifetimes, and large scattering samples are typically required. These reactions occur with secondary particles, i.e., the fast neutrons, and incident fluxes are low.

- Large amounts (>10 grams) of enriched isotopes are required in order to assure that the $\gamma$ rays are attributed to the correct nuclear state and that its properties are correctly determined. Large scattering samples are generally available on a lease arrangement from Oak Ridge National Laboratory, but the costs are high.

\subsubsection{Excitation functions}

By keeping the detection angle constant and varying the incident neutron energy, it is possible to build a level scheme. The $\gamma$-ray thresholds permit the placement of the emitting levels. These data complement the information obtained from other probes and from $\gamma-\gamma$ coincidence measurements. In addition, cross section data provide information about the angular momentum of the emitting levels.

\subsubsection{Angular distributions}

Gamma-ray angular distributions are produced by keeping the incident neutron energy constant and changing the detection angle and yield multipole mixing ratios, $\delta(\mathrm{E} 2 / \mathrm{M} 1)$, and 
level lifetimes from DSAM measurements. From these quantities, reduced transition probabilities are obtained.

\subsection{Experimental measurements}

To minimize the spectral contributions from other naturally occurring isotopes of Ge and Se and increase the sensitivity of the measurements, enriched targets $\left(19.56 \mathrm{~g}\right.$ of ${ }^{76} \mathrm{GeO}_{2}$ enriched to $84 \%$ and $11.96 \mathrm{~g}$ of elemental ${ }^{76} \mathrm{Se}$ powder enriched to $96.95 \%$ ) contained in polyethylene vials were used in the (n,n' $\gamma)$ measurements at UKAL. Gamma rays were detected in a high-purity germanium (HPGe) detector of $50 \%$ relative efficiency and an energy resolution of less than $2.2 \mathrm{keV}(\mathrm{FWHM})$ at $1333 \mathrm{keV}$ surrounded by a bismuth germanate (BGO) annulus. Neutrons with energies up to $3.7 \mathrm{MeV}$ were used in the excitation function measurements, and angular distributions were measured at $\mathrm{E}_{\mathrm{n}}=3.0$ and $3.5 \mathrm{MeV}$ for ${ }^{76} \mathrm{Ge}$ and at $2.4,3.0$, and $3.7 \mathrm{MeV}$ for ${ }^{76} \mathrm{Se}$. For each nucleus, level spins, level lifetimes, $\gamma$-ray branchings, and multipole mixing ratios were determined. In ${ }^{76} \mathrm{Ge}$, many previously suggested levels were found to have been placed in error and were eliminated. This issue proved to be less problematic in ${ }^{76} \mathrm{Se}$. The detailed results for ${ }^{76} \mathrm{Ge}$ have been published [11] and the results of the study of ${ }^{76} \mathrm{Se}$ will soon be submitted [12].

\section{Results and discussion}

A brief examination of the level structures of the ground bands (see Fig. 1) in these nuclei suggests that ${ }^{76} \mathrm{Se}$ is slightly more deformed and more collective than ${ }^{76} \mathrm{Ge}$. As noted earlier, nuclei in this region exhibit an array of structural features, including shape coexistence [4]. While shell model calculations reproduced the low-lying level structure of ${ }^{76} \mathrm{Ge}$ very well, they have not met with similar success in ${ }^{76} \mathrm{Se}$ and are ongoing [12].

An obvious structural feature in the data (see Figs. 2 and 3 ) is the collective band built on the lowest $0^{+}$excitation in ${ }^{76} \mathrm{Se}$ (see Fig. 3), which occurs nearly $1 \mathrm{MeV}$ lower in energy than in ${ }^{76} \mathrm{Ge}$ (see Fig. 2). Interestingly, the calculations by Nomura et al. [13] within mapped interacting boson model calculations based on the Gogny energy density functional predicts this state to lie even lower in excitation energy.
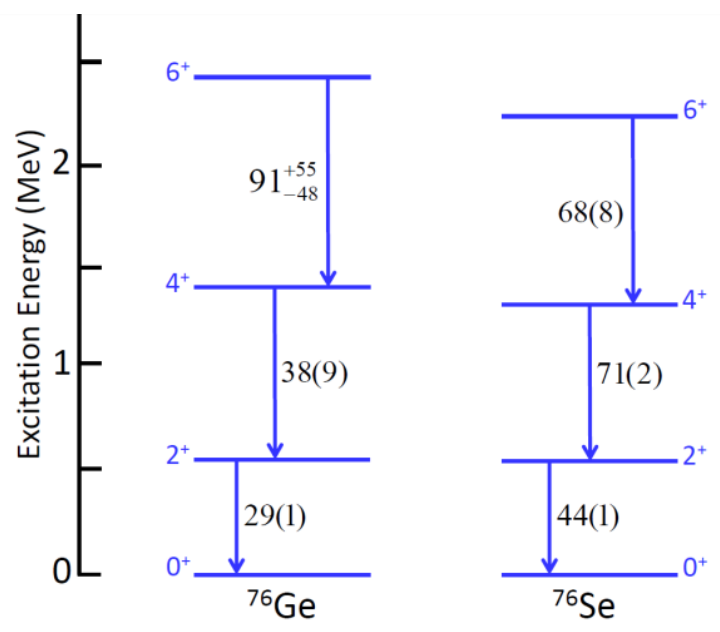

Fig. 1. Ground bands in ${ }^{76} \mathrm{Ge}$ and ${ }^{76} \mathrm{Se}$. B(E2) values on the transition arrows, in W.u., are from ENSDF [14], except for the $\mathrm{B}\left(\mathrm{E} 2 ; 6^{+} \rightarrow 4^{+}\right)$in ${ }^{76} \mathrm{Ge}$, which is from the current measurements. 


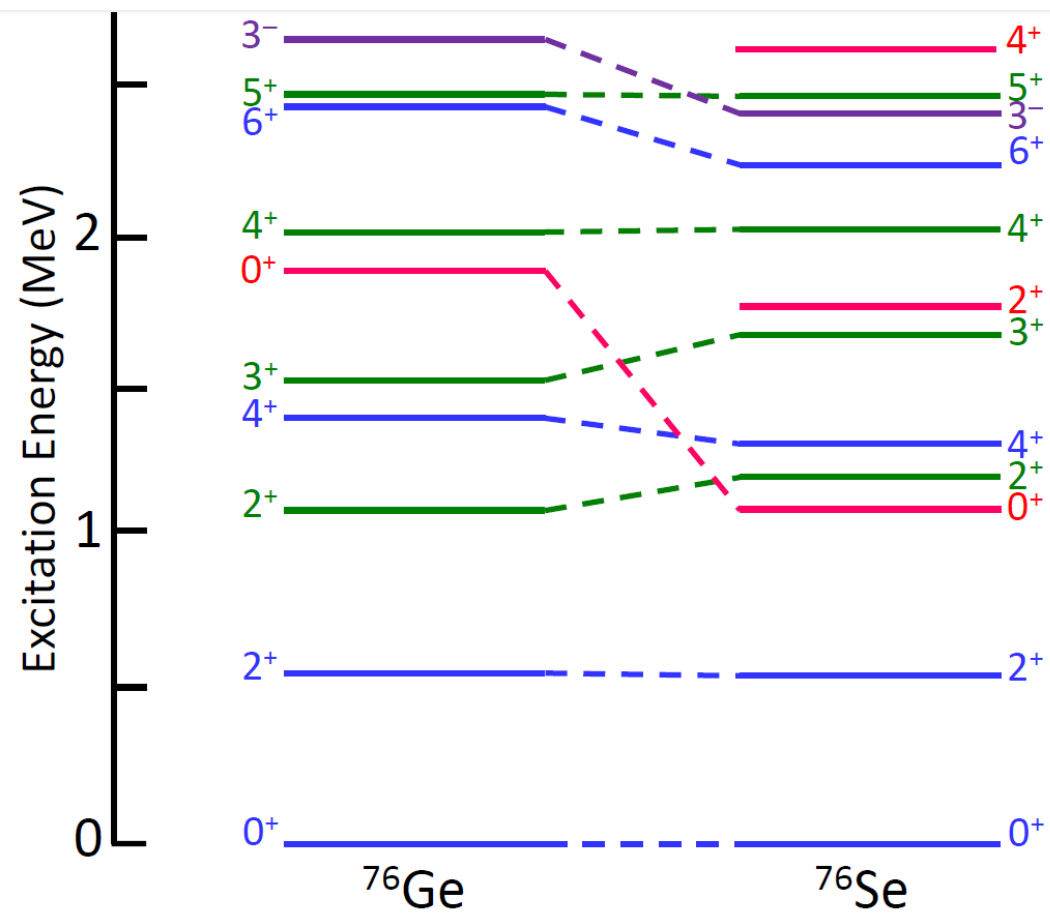

Fig. 2. Comparison of the level structures of ${ }^{76} \mathrm{Ge}$ and ${ }^{76} \mathrm{Se}$. Band members are shown in the same color.

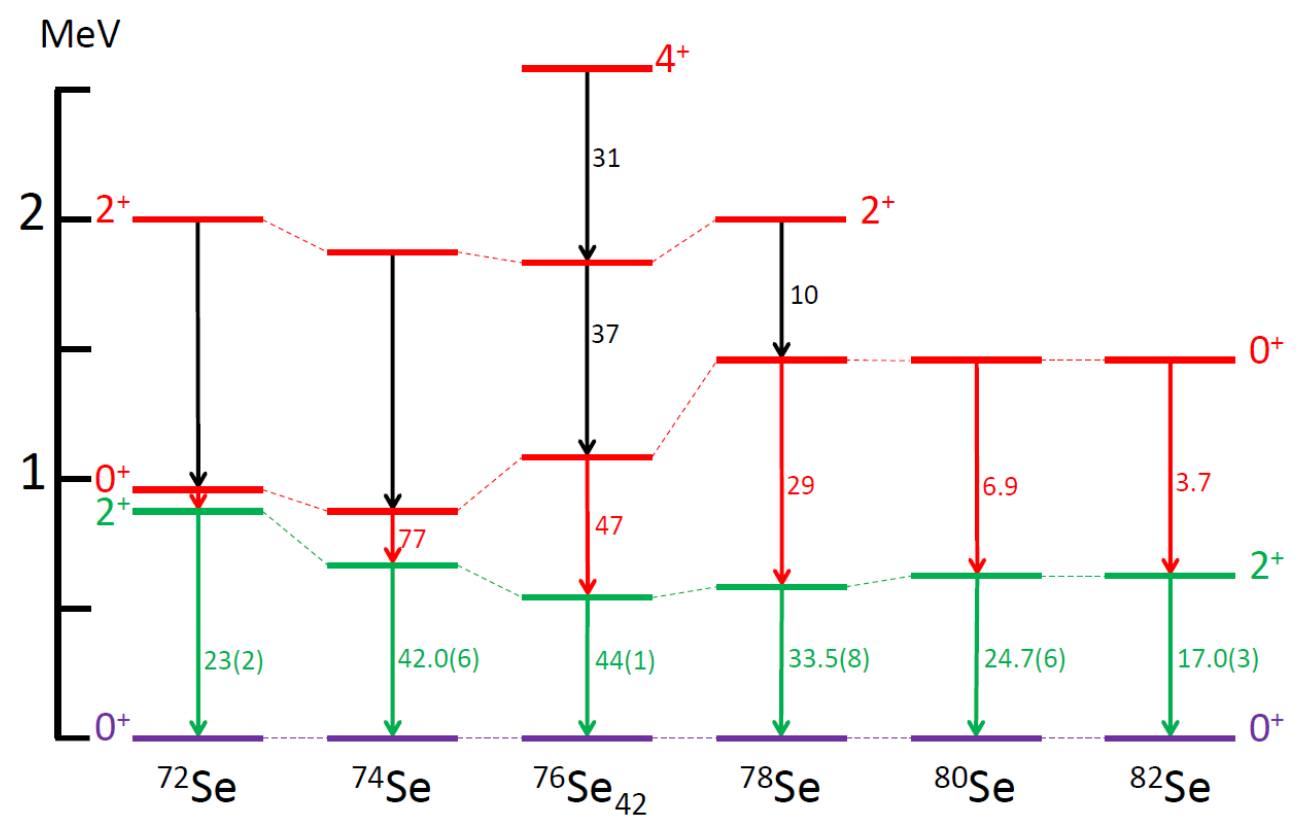

Fig. 3. Level systematics of the even-A Se nuclei. Except for the low-lying $0^{+}$band in ${ }^{76} \mathrm{Se}, \mathrm{B}(\mathrm{E} 2)$ values for the indicated transitions, given in W.u., were obtained from ENSDF [14]. The $2^{+}$and $4^{+}$ states of this band were identified in the current measurements. 
As in ${ }^{76} \mathrm{Ge}$, the low energy of the second excited $2^{+}$state, the band head of a quasi- $\gamma$ band, suggests triaxiality in ${ }^{76} \mathrm{Se}$. The calculations of Nomura et al. [13] describe the quasi$\gamma$ band in ${ }^{76}$ Se reasonably well and the $3^{+}$and $4^{+}$levels are close in energy, following the systematics of the $\gamma$-unstable-rotor model of Wilets and Jean [15].

\section{Summary}

The stable $\mathrm{A}=76$ nuclei, ${ }^{76} \mathrm{Ge}$ and ${ }^{76} \mathrm{Se}$, have been studied with the inelastic neutron scattering reaction. While there are clearly similarities between these nuclei, it may be that their deformations are considerably different, as suggested by Nomura et al. [13], and a recent theoretical calculation indicates that a transition from prolate ${ }^{76} \mathrm{Ge}$ to oblate ${ }^{76} \mathrm{Se}$ occurs [13]. The impact of this finding on the exotic decay process, $0 v \beta \beta$, remains to be evaluated.

We sincerely thank H.E. Baber and other colleagues at the UKAL for their contributions to these experiments. This material is based upon work supported by the U.S. National Science Foundation under Grant. No. PHY-1606890.

\section{References}

1. F.T. Avignone, S.R. Elliott, J. Engel, Rev. Mod. Phys. 80, 481 (2008)

2. J. Engel, J. Menéndez, Rep. Prog. Phys. 80, 046301 (2017)

3. Z. Podolyák, et al., Int. J. Mod. Phys. E 13, 123 (2004)

4. K. Heyde and J.L. Wood, Rev. Mod. Phys. 83, 1467 (2011)

5. Y. Toh, et al., Phys. Rev. C 87, 041304(R), (2013)

6. J.J. Sun, et al., Phys. Lett. B 734, 308 (2014)

7. T. Belgya, G. Molnár, S.W. Yates, Nucl. Phys. A607, 43 (1996)

8. E.E. Peters, et al., Phys. Rev. C 88, 024317 (2013)

9. C.A. McGrath, et al., Nucl. Instrum. Meth. A421, 458 (1999)

10. E. Elhami, et al., Phys. Rev. C 78, 064303 (2008)

11. S. Mukhopadhyay, et al., Phys. Rev. C 95, 014327 (2017)

12. S. Mukhopadhyay, et al., Phys. Rev. C, to be submitted (2018)

13. R. Nomura, K. Rodríguez-Guzmán, L.M. Robledo, Phys. Rev. C 95, 064310 (2017)

14. ENSDF, NNDC Online Data Service, ENSDF data base, http://www.nndc.bnl.gov/ensdf/ (2017)

15. L. Wilets and M. Jean, Phys. Rev. 102, 788 (1956). 